\title{
Potential target for mitigation of COVID-19 by food-derived bioactive peptides
}

\begin{abstract}
Kenji Sato
Division of Applied Biosciences, Graduate School of Agriculture, Kyoto University, Kitashirakawa Oiwakecho, Kyoto 6068502 , Japan. Tel: +81 75753 6444; Fax: +81 75753 6400; E-mail; sato.kenji.7x@kyoto-u.ac.jp

DOI: $10.31665 /$ JFB.2021.16287

Received: October 02, 2021; Revised received \& accepted: November 16, 2021

Citation: Sato, K. (2021). Potential target for mitigation of COVID-19 by food-derived bioactive peptides. J. Food Bioact. 16: 3-7.

Abstract

Oral administration of food protein hydrolysate and naturally occurring peptides exert beneficial effects beyond conventional nutritional functions by supplying amino acids for protein synthesis. These peptides are referred to as food-derived bioactive peptides. The coronavirus disease 2019 (COVID-19) is caused by sever acute respiratory syndrome coronavirus 2 (SARS-CoV-2) infection. Some host and viral proteins are involved in the entry of SARSCoV-2 into cells and their replication. Peptides with specific sequences can interact with these proteins and have potential prophylactic and therapeutic activities against COVID-19. However, it is difficult to deliver food-derived peptides to target organs without degradation by exopeptidases in the body. Alternatively, food-derived peptides and amino acid metabolites have been suggested to decrease risk factors of COVID-19 by modulating the reninangiotensin system, the innate immune system, and the antioxidant system. This mini-review is based on in vivo responses to food-derived peptides and aims to introduce potential targets for these peptides in decreasing the risk and severity of COVID-19.
\end{abstract}

Keywords: Food peptide; COVID-19; Angiotensin converting enzyme; Antimicrobial peptide; Glutathione.

\section{Introduction}

Coronavirus disease 2019 (COVID-19) is an infectious disease caused by the severe acute respiratory syndrome coronavirus 2 (SARS-CoV-2). The COVID-19 pandemic has become a serious threat to human health. Till date, existing drugs, including anti-inflammatory drugs, such as synthetic corticosteroides and monoclonal antibodies against proinflammatory cytokines and their receptors, such as interleukin- 6 and its receptor, and antiviral drugs based on inhibition of proteases, RNA polymerases, reverse transcriptases that are necessary for viral duplication, have been evaluated for their efficacy against COVID-19 (Harrison, 2020). FDA and other Ministries have approved some of them for clinical use for COVID-19 patients. Furthermore, many efforts have been made to elucidate the nature of SARS-CoV-2 and related viruses. Consequently, some SARS-CoV-2 and host proteins responsible for virus entry into human cells have been identified (Coutard et al., 2020; Hoffman et al., 2020; Wrapp et al., 2020). Newly gained insight about the mechanism of the viral infection provides new drug targets for
SARS-CoV-2 infection. Currently, vast efforts are being focused on developing anti-COVID-19 drugs based on the mechanism of viral entry into human cells. Monoclonal antibodies against SARS-CoV-2 surface spike proteins (Chen et al., 2021) and inhibitors of proteases, which are involved in the priming and maturation of SARS-CoV-2 spike protein (Schütz et al., 2020), are being developed.

Clinically, SARS-CoV-2 infection exhibits a wide range of symptoms, ranging from asymptomatic to mild respiratory illness to deadly acute respiratory distress (Wu and McGoogan, 2020). Many risk factors, such as increasing age, hypertension, ischemic heart disease, diabetes, chronic respiratory disease, obesity, preexisting malignancy, and smoking have been reported. From these multiple and heterogeneous risk factors, a common pathway that can increase the severity and mortality rates of COVID-19 has been identified. It has been suggested that oxidative stress caused by unregulated inflammation, also known as a "cytokine storm," plays a significant role in increasing the severity of COVID-19 (Chen et al., 2020). Depression of the host antioxidant enzyme system because of depletion of glutathione has been suggested to 
Table 1. Potential targets for food-derived peptides and related compounds to decrease potential risk of COVID-19

\begin{tabular}{|c|c|c|c|}
\hline Targets & Action & $\begin{array}{l}\text { Potential active peptides } \\
\text { and related compounds }\end{array}$ & $\begin{array}{l}\text { References on ac- } \\
\text { tive compounds* }\end{array}$ \\
\hline $\begin{array}{l}\text { Angiotensin converting } \\
\text { enzyme ( } A C E)\end{array}$ & $\begin{array}{l}\text { Suppression of angiotensin II-induced } \\
\text { inflammation and hypertension }\end{array}$ & $\begin{array}{l}\text { Val-Tyr; } \\
\text { Ile-Pro-Pro }\end{array}$ & $\begin{array}{l}\text { Matsui et al., 2002; } \\
\text { Dong et al., } 2013\end{array}$ \\
\hline $\begin{array}{l}\text { Type II transmembrane } \\
\text { serine proteases (TMPRSS2) }\end{array}$ & Inhibition of SARS-CoV-2 entry into cell & Concept only & \\
\hline Furin & Inhibition of SARS-CoV-2replication in cell & Concept only & \\
\hline Host antiviral peptide & $\begin{array}{l}\text { Enhance host anti-viral peptides ( } \alpha \text {-defensin); } \\
\text { Enhance host anti-viral peptides ( } \beta \text {-defensin); } \\
\text { Enhance host anti-viral peptides lactoferrin }\end{array}$ & $\begin{array}{l}\text { pyroGlu-Leu; } \\
\text { Concept only; } \\
\text { Concept only }\end{array}$ & Shirako et al., 2019; \\
\hline Glutathione depletion & $\begin{array}{l}\text { Supplying glutathione; Supplying precursor } \\
\text { of glutathione; Retaining hepatic cysteine } \\
\text { by suppressing generation of aldehydes }\end{array}$ & $\begin{array}{l}\text { Glutathione; } \\
\text { N-acetyl-cysteine; } \\
\text { Phenethylamine }\end{array}$ & $\begin{array}{l}\text { Yamada et al., 2018; } \\
\text { Yamada et al., 2018; } \\
\text { Zheng et al., } 2021\end{array}$ \\
\hline
\end{tabular}

*These studies have not demonstrated direct action on COVID-19, but demonstrated the suggested actions.

be responsible for the deadly increase in oxidative stress in COVID-19 patients (Khanfar and Qaroot, 2020; Polonikov, 2020; Silvagno et al., 2020). This hypothesis may provide a new target for the mitigation of the disease state of in COVID-19 patients.

As mentioned above, considerable effort has been focused on the development of drugs against COVID-19. In addition, dietary approaches and the use of supplements may play a positive role in mitigating the severity of the disease. The potential targets of foodderived peptides and related compounds for reducing the risk of SARS-CoV-2 infection and mitigating the severity of COVID-19 symptoms are discussed in the following sections.

\section{Food-derived bioactive peptides}

Peptides are short chains of amino acids usually linked by peptide bonds, normally between $\alpha$-amino/imino groups and $\alpha$-carboxyl groups; some peptides can have non- $\alpha$ peptide bonds. Peptides can be prepared by partial hydrolysis of proteins and chemical synthesis. In addition, some naturally occurring non-ribosomal peptides, such as imidazole peptides and glutathione, are present in bacteria, fungi, yeast, plants, and animals. Enzymatic hydrolysates of food proteins are the most commonly used food ingredients. Naturally occurring non-ribosomal peptides, such as glutathione and imidazole peptides, are produced by fermentation and hot water extraction of fish and chicken, respectively, and are used as food ingredients (Toldrá et al., 2018). In a fewer cases, chemically synthetic peptides are used as food additives, such as asparatame (Yokozeki and Abe, 2021). While chemical synthesis can add non-naturally occurring amino acids and unique chemical groups into the peptides, and thus increase the bioavailability and efficacy of peptidebased drugs, this review chose to discuss the potential prophylactic and therapeutic applications of food-derived peptides rather than peptide-based drugs. Proposed targets of food-derived peptides and potential actions are summarized in Table 1 .

\section{Targets for food-derived peptides}

\subsection{Host proteinase and peptidase}

SARS-CoV-2 binds angiotensin-converting enzyme 2 (ACE2) on the surface of human cells with the viral spike protein (S protein) in order to enter cells (Hoffmann et al., 2020). The SARS-CoV-2 $\mathrm{S}$ protein consists of two subunits, S1 and S2. Binding of the S protein to ACE2 induces conformal changes in the $\mathrm{S}$ protein, which permits host type II transmembrane serine proteases (TMPRSS2) to undergo proteolytic processing of the viral S2 subunit. This priming process of the $\mathrm{S}$ protein is vital for viral and host cell membrane fusion (Wrapp et al., 2020). After viral entry into host cells, the newly synthesized SARS-CoV-2 S protein is cleaved by host intracellular proteinases, such as furin, into $\mathrm{S} 1$ and $\mathrm{S} 2$ subunits, which possibly increases the infectivity of newly generated SARS-CoV-2 (Coutard et al., 2020). These facts indicate that the host renin-angiotensin-aldosterone system, TMPRSS2, and furin can be potential targets for the inhibition of SARS-CoV-2 replication (Table 1). The potential applications of food-derived bioactive peptides on these host proteases and peptidases were recently reviewed by Bhullar et al. (2021), in which the detailed mechanism for SARS-CoV-2 entry and replication in host cells and the structure of proteins involved are described. In this article, an outline of potential inhibition of host proteases by food-derived peptides is presented. In addition, some views on the bioavailability and the potential metabolic fate of bioactive peptides have been added.

Yoshikawa and Chiba have demonstrated that oral administration of food-derived peptides mitigates hypertension, which is one of the earliest achievements in functional food and nutraceutical areas (Yokoyama et al., 1992). It has been proposed that hypertension can be mitigated via the inhibition of angiotensin-converting enzyme (ACE) by bioactive peptides. ACE, a metallopeptidase, converts angiotensin I (decapeptide), which is generated from angiotensinogen by proteolytic cleavage with renin, into angiotensin II (octapeptide). Angiotensin II increases blood pressure and inflammatory response by binding to angiotensin II receptor 1 (ATR1), which is believed to worsen the disease progression of COVID-19. ACE2, a receptor for SARS-CoV-2 S protein, is a homolog of ACE (Boehm and Nabel, 2002). ACE2 converts angiotensin II (octapeptide) into angiotensin (1-7, heptapeptide) by releasing the carboxy terminal amino acid. Angiotensin (1-7) does not activate ATR1 and serves as a ligand for the Mas receptor. Angiotensin (1-7) has been demonstrated to be protective against angiotensin II-mediated pulmonary vasoconstriction and inflammation (Boehm and Nabel, 2002). Binding of SARS-CoV-2 to ACE2 decreases the available ACE2 to ACE ratio, which can increase angiotensin II levels and induce subsequent angiotensin IImediated events. Thus, blocking ACE2 by bioactive peptides may 
exacerbate COVID-19. Therefore, ACE and ATR1, rather than ACE2, may be good targets for the mitigation of COVID-19 (Bhullar et al., 2021). ACE inhibitors and ATR1 blockers are available as medicines. Some animal studies have suggested that ACE inhibitors and ATR1 blockers may alleviate COVID-19 progression but do not affect viral replication (Gao et al., 2021). Food-derived peptides with antihypertensive activity may exert similar effects. However, ACE inhibitory drugs should be used for the treatment of COVID-19 in the acute phase, as they exert stronger ACE inhibition in the body compared to that by food-derived peptides (Dong et al., 2013). However, some food-derived peptides have been demonstrated to suppress mild hypertension, a risk factor for COVID-19 (Matsui et al., 2002, Dong et al., 2013). Thus, foodderived peptides might be used to decrease the risk of COVID-19, although data to supporting this hypothesis is lacking.

Inhibition of other proteases, such as TMPRSS2 and furin, which are involved in the priming and maturation of SARS-CoV-2 $\mathrm{S}$ protein, are potential targets for food-derived peptides to inhibit viral entry into cells and the subsequent replication (Bhullar et al., 2021). In addition, the lysosomal protease, cathepsin L, is also suggested to be involved in the SARS-CoV spike priming (Huang et al., 2005). In vitro inhibitory assays can identify some peptides with inhibitory activities against these proteases from food protein hydrolysates as well as ACE inhibitory peptides. However, it has been demonstrated that the bioavailability of food-derived peptides with in vitro ACE activity is very low because of high susceptibility to exopeptidase digestion (Matsui et al., 2004; Foltz et al., 2007). Thus, chemically modified peptides have been synthesized to increase bioavailability. The peptide-based drugs are being evaluated for therapeutic effects against COVID-19 (Schuütz et al., 2020). Nevertheless, oral administration of some food-derived peptides decreased angiotensin II in the body, while its blood concentration was far lower than the concentration used in the in vitro assay (Matsui et al., 2004). These facts suggest that some metabolites or second messengers generated by administration of the peptides might inhibit ACE in the body. In addition, furin and cathep$\sin \mathrm{L}$ act on viral S protein in host cell. Thus, inhibitory peptides or its metabolites must be incorporated into target cells to inhibit viral replication. Taken together, these findings suggest that the in vitro inhibition of proteases cannot be directly associated with inhibition of viral replication by oral administration of food-derived peptides; however, it may be useful in designing peptide-based drugs as bioavailability can be improved by chemical modification.

\subsection{Host antimicrobial peptides}

Antimicrobial peptides (AMPs) are produced by a wide variety of eukaryotes as essential components of the innate immune response against pathogens (Ganz, 2002). AMPs generally have a net positive charge and are amphiphilic, which allows them to interact with the negatively charged molecules on the surfaces of bacteria, enveloped viruses, fungi, and cancer cells; they destabilize the surface membrane by creating transmembrane channels and kill the pathogens. Some AMPs have been demonstrated to exert virucidal activity against coronaviruses. Defensins are a family of AMPs that are involved in host defense. Human $\alpha$-defensin 5 (HDEF5) is an AMP secreted by Paneth cells in the small intestine and neutrophils. HDEF5 can attach to the SARS-CoV-2 S1 protein through the glycosylated residue of the protein (Niv, 2020). Mouse $\beta$-defensins- 4 derived peptide binds to the Middle East respiratory syndrome (MERS)-CoV S2 subunit (Mahendran et al., 2020). The $\beta$-defensins- 4 derived peptide inhibits priming of MERS-CoV S protein by cathepsin $\mathrm{L}$ in endosomes because of its high polyca- tionic property, even though it does not inhibit viral entry into the host cell (Zhao et al., 2016). Thus, it exerts both prophylactic and therapeutic effects in an in vivo SARS-CoV mouse model of infection (Zhao et al., 2016). Lactoferrin is an iron-binding glycoprotein that is present in the colostrum and several mucosal layers. It has been reported that lactoferrin interfered with virus adsorption by blocking the binding of the spike proteins of SARS-CoV and SARS-CoV-2 to host cell receptors (Lang et al., 2011; Elnagdy and AlKhazindar, 2020). Histones are highly basic proteins that are found in eukaryotic cell nuclei. Histones and their fragment peptides are also found in cytosol and extracellular fluids and exert antimicrobial and antiviral activities. It has been demonstrated that histones are able to neutralize influenza A viruses (Hoeksema et al., 2015). It has been speculated that the aerosol spray of recombinant AMPs with antiviral activity to the upper airway has prophylactic and therapeutic potential against COVID-19. Some food-derived peptides exhibit antimicrobial activity (Pellegrini, 2003). Even if antiviral activity is present in food-derived peptides such as lactoferrin, it is, however, difficult to deliver them with anti-viral activity to respiratory tracts by oral administration. Oral administration of the small food-derived peptide, pyroglutamyl-leucine (pyroGlu-Leu), which was found in enzymatic hydrolysates of wheat gluten, corn gluten, and fermented foods, can increase intestinal $\alpha$-defensin-related AMP, rattusin, in an animal model (Shirako et al., 2019). An increase in pyroGlu-Leu levels in the blood flow after oral ingestion was observed (Wada et al., 2013). Thus, it is possible that ingestion of some food-derived peptides might increase host AMPs in the mucosal layer of the respiratory tract as well as in the intestinal tract. Thus, modulation of host AMP secretion rather than direct antiviral activity may be a good target for food-derived peptides (Table 1).

\subsection{Glutathione}

As mentioned above, the majority (approximately $80 \%$ ) of people infected with SARS-CoV-2 are asymptomatic, which indicates the presence of risk factors for exacerbation of COVID-19 (Chen et al., 2020; Wu and McGoogan, 2020). Studies have suggested many COVID-19 risk factors, including increasing age, hypertension, ischemic heart disease, diabetes, chronic respiratory disease, obesity, pre-existing malignancy, and smoking. It has been hypothesized that these heterogeneous risk factors could be associated with a common pathway that exacerbates COVID-19 mortality. It has been shown that COVID-19-related mortality is highly associated with the so-called "cytokine storm" induced by the virus (Ragab et al., 2020). SARS-CoV-2 RNA acts as a pathogen-associated molecular pattern recognized by toll-like receptors. As mentioned above, SARS-CoV-2 S protein blocks ACE2, which also increases the inflammatory response via the angiotensin II-ATR1 axis. Therefore, rapid viral replication can induce pro-inflammatory cytokines and chemokines, consequently inducing a cytokine storm. Thus, anti-inflammatory drugs, such as dexamethasone, can prove useful in the treatment of COVID-19 (Horby, et al., 2021). However, their efficacy is limited. Currently, monoclonal antibodies against interleukin (IL)-6 and its receptor are used, as IL-6 plays crucial role in cytokine storm (Harrison, 2020). It has been demonstrated that some food-derived peptides mitigated chronic inflammation (Majumder et al., 2016). Oral administration of ValPro-Tyr (100 mg/kg body weight) was demonstrated to alleviate experimental colitis in mouse model and decrease gene expression of the pro-inflammatory cytokines TNF- $\alpha$, IL- 6 , IL- $1 \beta$, IFN- $\gamma$, and IL-17 in the colon (Kovacs-Nolan et al., 2012), which indicates that some food-derived peptide exert anti-inflammatory activity. 
However, effect of food-derived peptide on acute and sever inflammation remains to be examined. Immune cells activated by proinflammatory cytokines can generate reactive oxygen species (ROS), which cause oxidative stress and tissue injury. Oxidative stress resulting from an increased production of ROS and/or decreased antioxidant defense system has been demonstrated to contribute to the pathogenesis of various chronic diseases that are risk factors for COVID-19 (Pincemail et al., 2021). Metabolome analyses of the blood have suggested that high oxidative stress markers and low blood glutathione levels are common factors for patients in the high-risk group for COVID-19 (Khanfar and Qaroot, 2020; Polonikov, 2020; Pincemail et al., 2021). Furthermore, patients with moderate and severe COVID-19 illness had lower levels of glutathione and higher ROS and ROS/glutathione ratio in plasma than patients with mild disease, indicating that glutathione deficiency and oxidative stress are associated with the severity of COVID-19. Moreover, glutathione depletion triggers the apoptosis of lymphocytes, which is associated with a high mortality rate in COVID-19 patients.

Glutathione is an endogenous peptide with a sequence of $\gamma$-glutamyl-cysteinyl-glycine. It serves as a co-enzyme and substrate for glutathione peroxidase and glutathione S-transferase, respectively, which are crucial enzymes for antioxidant and detoxification in cells (Silvagno et al., 2020). Glutathione peroxidase reduces hydrogen peroxide and lipid peroxidation by using glutathione. Glutathione is then converted to glutathione disulfide, an oxidative form of glutathione. Thus, the glutathione/glutathione disulfide ratio can be used as a redox indicator in the body. The oxidized form of glutathione, glutathione disulfide, is converted to its reduced form by glutathione reductase. In this enzymatic system, the reduced form of nicotinamide adenine dinucleotide phosphate (NADPH) is used for the reduction of glutathione disulfide, which can be supplied by glucose metabolism in the pentose phosphate pathway. Glutathione can be synthesized from three constituent amino acids by glutamate cysteine ligase and glutathione synthase. These processes help in maintaining the cellular level of glutathione (Silvagno et al., 2020). Nevertheless, glutathione depletion has been observed in patients at high risk of COVID-19 and with severe COVID-19 (Khanfar and Qaroot, 2020; Polonikov, 2020; Pincemail et al., 2021). It has been assumed that the overproduction of ROS can lead to glutathione depletion. Thus, clinical trials using supplementation of glutathione and its precursor, $\mathrm{N}$-acetylcysteine (NAC), in patients with COVID-19 have been initiated. Some preliminary studies suggested that the administration of NAC exhibited positive results (Cazzola et al., 2021). It has been demonstrated that orally administered stable isotope-labeled glutathione is directly absorbed into the bloodstream and incorporated into the liver, while glutathione levels in the liver do not change following oral administration of glutathione under normal conditions in rats (Yamada et al., 2018). Orally administered NAC is also incorporated into the liver and converted to glutathione in rats (Yamada et al., 2018). Therefore, supplementation of glutathione and NAC in patients with endogenous glutathione depletion could mitigate glutathione depletion and COVID-19 (Table 1). However, there is limited data on pulmonary glutathione levels in COVID-19 patients with different severities.

As mentioned above, it has been assumed that high levels of ROS directly induce blood glutathione depletion, while glutathione can be synthesized and its reduced form is maintained by enzymatic systems. These facts suggest that these enzymatic systems might be suppressed in the patients with high-risk factors and with severe COVID-19; this remains to be examined further. Ironically, an in vitro study demonstrated that dexamethasone decreased glutathione levels in human alveolar epithelial cells (A549) by de- creasing glutamate cysteine ligase activity (Rahman et al., 1998), which could explain the limited efficacy of dexamethasone for the treatment of COVID-19. It has been demonstrated that shortchain aldehydes, such as malondialdehyde, methylglyoxal, and acetaldehyde react with sulfanyl groups of cysteine and cysteinyl residues in peptides and proteins. These short-chain aldehydes can be generated by oxidation of lipids and by metabolism of sugar and alcohol, respectively. Recently, severe depletion of cysteine, a precursor of glutathione, was found in the liver of high-fat dietinduced obese mice, while the mice received sufficient levels of cystine from their diet (Zheng et al., 2021). Increased generation of methylglyoxal possibly due to disorder of glucose metabolism (decrease of glyceraldehyde-3 phosphate dehydrogenase, GAPDH) by high-fat diet feeding and subsequent generation of malondialdehyde via oxidation of lipids have been suggested to be associated with the depletion of cysteine. These facts suggest that glucose metabolic disorder can increase reactive short-chain aldehydes and deplete cellular cysteine, which might decrease cellular glutathione levels and increase the risk and severity of COVID- 19. This hypothesis potentially explains the low glutathione levels in patients with chronic diseases. Further studies are necessary to confirm this hypothesis.

Numerous studies have demonstrated that some food-derived peptides exhibit antioxidant activity in vitro, based on which its potent antioxidant effect in the body has been suggested (Pan et al., 2020). However, sufficient levels of food-derived antioxidant peptides in the body have not been demonstrated. Nevertheless, oral administration of some peptide preparations and related compounds increases body glutathione levels, however, the underlying mechanism remains unclear. Recently, it has been demonstrated that oral administration of trace levels $(10 \mu \mathrm{g} / \mathrm{kg}$ body weight $)$ of phenethylamine, (decarboxylated phenylalanine), mitigated highfat diet-induced depletion of cysteine in mouse liver by decreasing reactive short-chain aldehydes via suppression of high-fat dietinduced decrease of GAPDH levels (Zheng et al., 2021). These facts indicate that oral administration of peptides and amino acid metabolites has the potential to increase cellular cysteine levels, which are precursors of glutathione, by improving the metabolism of sugar. Thus, direct supplementation of glutathione and its precursor, and suppression of excess generation of short-chain aldehydes, which can deplete cysteine, by food-derived peptides and amino acid metabolites, could maintain cellular glutathione levels and decrease the risk of COVID-19 severity.

\section{Conclusion}

After the outbreak of COVID-19, considerable parts of the life cycle of SARS-CoV-2 have been elucidated in a short time. This information provides drug targets for peptide-based medicine, as peptides with specific sequences can bind host and viral proteins and inhibit replication of the virus. However, it is difficult to deliver food-derived peptides with biological activities detected by in vitro assays to target organs because of their susceptibility to exopeptidase. Nevertheless, it has been demonstrated that oral administration of specific food-derived peptides and related compounds decreases angiotensin II, and increases host antimicrobial peptides and suppresses the decrease of glutathione and its precursor. These facts suggest that food-derived peptides have the potential to decrease the risk factors for COVID-19. However, their mechanisms remain to be elucidated. A deeper understanding of these mechanisms would be helpful in managing the risk factors of COVID-19. 


\section{References}

Bhullar, K.S., Drews, S.J., and Wu, J. (2021). Translating bioactive peptides for COVID-19 therapy. Eur. J. Pharmacol. 890: 173661.

Boehm, M., and Nabel, E.G. (2002). Angiotensin-converting enzyme 2-a new cardiac regulator. N. Engl. J. Med. 34: 1795-1797.

Cazzola, M., Rogliani, P., Salvi, S.-S., Ora, J., and Matera, M.G. (2021). Use of thiols in the treatment of COVID-19: Current evidence. Lung 199: 335-343.

Chen, J., Gao, K., Wang, R., Nguyen, D.D., and Wei, G.-W. (2021). Review of COVID-19 antibody therapies. Annu. Rev. Biophys. 50: 1-30.

Chen, N., Zhou, M., Dong, X., Qu, J., Gong, F., Han, Y., Qiu, Y., Wang, J., Liu, Y., and Wei, Y. (2020). Epidemiological and clinical characteristics of 99 cases of 2019 novel coronavirus pneumonia in Wuhan, China: a descriptive study. Lancet 395: 507-513.

Coutard, B., Valle, C., de Lamballerie, X., Canard, B., Seidah, N., and Decroly, E. (2020). The spike glycoprotein of the new coronavirus 2019$\mathrm{nCoV}$ contains a furin-like cleavage site absent in CoV of the same clade. Antivir. Res. 176: 104742.

Dong, J.-Y., Szeto, I.M.Y., Makinen, K., Gao, Q., Wang, J., Qin, L.-Q., and Zhao, Y. (2013). Effect of probiotic fermented milk on blood pressure: a metaanalysis of randomised controlled trials. Brit. J. Nutri.110: 1188-1194.

Elnagdy, S., and AlKhazindar, M. (2020). The potential of antimicrobial peptides as an antiviral therapy against COVID-19. ACS Pharmacol. Transl. Sci. 3: 780-782.

Foltz, M., Meynen, E.E., Bianco, V., Platerink, C., van Koning, T.M.M.G., and Kloek, J. (2007). Angiotensin converting enzyme inhibitory peptides from a lactotripeptide-enriched milk beverage are absorbed intact into the circulation. J. Nutri. 137: 953-958.

Ganz, T. (2002). Epithelia: Not just physical barriers. PNAS 99: 3357-3358.

Gao, W.-C., Ma, X., Wang, P., He, X.-Y., Zheng, Y.-T., Liu, F.-L., and Zheng, C.-B. (2021). Captopril alleviates lung inflammation in SARS-CoV-2-infected hypertensive mice. Zool Res. 42: 633-636.

Harrison, C. (2020). Coronavirus puts drug repurposing on the fast track. Nature Biotech. 38: 379-391.

Hoeksema, M., Tripathi, S., White, M., Qi, L., Taubenberger, J., van Eijk, M., Haagsman, H., and Hartshorn, K.L. (2015). Arginine-rich histones have strong antiviral activity for influenza A viruses. Innate Immun. 21: 736-745.

Hoffmann, M., Kleine-Weber, H., Schroeder, S., Krüger, N., Herrler, T., Erichsen, S., Schiergens, T.S., Herrler, G., Wu, N.-H., Nitsche, A., Müller, M., Drosten, C., and Pöhlman, S. (2020). SARS-CoV-2 cell entry depends on ACE2 and TMPRSS2 and is blocked by a clinically proven protease inhibitor. Cell 181: 271-280.

Horby, P., Lim, W.S., Emberson, J.R., Mafham, M., Bell, J.L., Linsell, L., Staplin, N., Brightling, C., Ustianowski, A., Elmahi, E., Prudon, B., Green, C., Felton, T., Chadwick, D., Rege, K., Fegan, C., Chappell, L.C., Faust, S.N., Jaki, T., Jeffery, K., Montgomery, A., Rowan, K., Juszczak, E., Baillie, J.K., Haynes, R., and Landray, M.J. (2021). Dexamethasone in Hospitalized Patients with Covid-19. N. Engl. J. Med. 384: 693-704.

Huang, I.-C., Bosch, B.J., Li, F., Li, W., Lee, K.H., Ghiran, S., Vasilieva, N., Dermody, T.S., Harrison, S.C., Dormitzer, P.R., Dermody, T.S., Harrison, S.C., Dormitzer, P.R., Farzan, M., Rottier, P.J.M., and Choe, H. (2005). SARS coronavirus, but not human coronavirus NL63, utilizes cathep$\sin$ L to infect ACE2-expressing cells. J. Boil. Chem. 281: 3198-3203.

Khanfar, A., and Qaroot, B.A.L. (2020). Could glutathione depletion be the Trojan horse of COVID-19 mortality? Eur. Rev. Med. Pharmacol. Sci. 24: $12500-12509$

Kovacs-Nolan, J., Zhang, H., Ibuki, M., Nakamori, T., Yoshiura, K., Turner P.V., Matsui, T., and Mine, Y. (2012). The PepT1-transportable soy tripeptide VPY reduces intestinal inflammation. Bioch. Biophys. Acta 1820: 1753-1763.

Lang, J., Yang, N., Deng, J., Liu, K., Yang, P., Zhang, G., and Jiang, C. (2011). Inhibition of SARS pseudovirus cell entry by lactoferrin binding to heparan sulfate proteoglycans. PLoS One 6(8): e23710.

Mahendran, A.S.K., Lim, Y.S., Fang, C.-M., Hwei-San Loh, H.-S., and Le, C.F. (2020). The potential of antiviral peptides as COVID-19 therapeutics. Front. Pharmacol. 11: 575444

Majumder, K., Mine, Y., and Wu, J. (2016). The potential of food proteinderived anti-inflammatory peptides against various chronic inflam- matory diseases. J. Sci. Food Agric. 96: 2303-2311

Matsui, T., Imamura, M., Oka, H., Osajima, K., Kimoto, K., Kawasaki, T., and Matsumoto, K. (2004). Tissue distribution of antihypertensive dipeptide, Val-Tyr, after its single oral administration to spontaneously hypertensive rats. J. Pept. Sci. 10: 535-545.

Matsui, T., Tanaka, K., Seki, E., Osajima, K., Matsumoto, K., and Kawasaki, T. (2002). Absorption of Val-Tyr with in vitro angiotensin I-converting enzyme inhibitory activity into the circulating blood system of mild hypertensive subjects. Biol. Pharm. Bull. 25: 1228-1230.

Niv, Y. (2020). Defensin 5 for prevention of SARS-CoV-2 invasion and Covid-19 disease. Med. Hypotheses 143: 110244.

Pan, M., Liu, K., Yang, J., Liu, S., Wang, S., and Wang, S. (2020). Advances on food-derived peptic antioxidants-A review. Antioxidant 9: 799.

Pellegrini, A. (2003). Antimicrobial peptides from food proteins. Curr. Pharm. Des. 9: 1225-38.

Pincemail, J., Cavalier, E., Charlier, C., Cheramy-Bien, J.-P., Brevers, E., Courtois, A., Fadeur, M., Meziane, S., Le Goff, C., Misset, B., Albert, A., Defraigne, J.-O., and Anne-Françoise Rousseau, A.-F. (2021). Oxidative stress status in COVID-19 patients hospitalized in intensive care unit for severe pneumonia. A pilot study. Antioxidant 10(2): 257.

Polonikov, A. (2020). Endogenous deficiency of glutathione as the most likely cause of serious manifestations and death in COVID-19 patients. ACS Infect. Dis. 6(7): 1558-1562.

Ragab, D., Eldin, H.S., Taeimah, M., Khattab, R., and Salem, R. (2020). The COVID-19 cytokine storm; What we know so far. Front. Immunol. 11 1446.

Rahman, I., Bel, A., Mulier, B., Donaldson, K., and MacNee, W. (1998). Differential regulation of glutathione by oxidants and dexamethasone in alveolar epithelial cells. Am. J. Physiol. 275: L80-6.

Schuütz, D., Ruiz-Blanco, Y.B., Muünch, J., Kirchhoff, F., Sanchez-Garcia, E., and Muüller, J.A. (2020). Peptide and peptide-based inhibitors of SARS-CoV-2 entry. Adv. Drug Delivery Rev. 167: 47-65.

Shirako, S., Kojima, Y., Tomari, N., Nakamura, Y., Matsumura, Y., Ikeda, K., Inagaki, N., and Sato, K. (2019). Pyroglutamyl leucine, a peptide in fermented foods, attenuates dysbiosis by increasing host antimicrobial peptide. NPJ Sci. Food 3: 18

Silvagno, F., Vernone, S., and Pescarmona, G.P. (2020). The role of glutathione in protecting against the severe inflammatory response triggered by COVID-19. Antioxidant 9: 624 .

Toldrá, F., Reig, M., Aristoy, M.-C., and Mora, L. (2018). Generation of bioactive peptides during food processing. Food Chem. 267: 395-404.

Wada, S., Sato, K., Ohta, R., Wada, E., Bou, Y., Fujiwara, M., Kiyono, T., Park, E.Y., Aoi, W., Takagi, T., Naito, Y., and Yoshikawa, T. (2013). Ingestion of low dose pyroglutamyl leucine improves dextran sulfate sodiuminduced colitis and intestinal microbiota in mice. J. Agric. Food Chem. 61: 8807-8813.

Wrapp, D., Wang, N., Corbett, K.S., Goldsmith, J.A., Hsieh, C.-L., Abiona, O., Graham, B.S., and McLellan, J.S. (2020). Cryo-EM structure of the 2019nCoV spike in the prefusion conformation. Science 367: 1260-1263.

Wu, Z., and McGoogan, J.M. (2020). Characteristics of and important lessons from the coronavirus disease 2019 (COVID-19) outbreak in China: Summary of a report of 72,314 cases from the Chinese Center for Disease Control and Prevention. JAMA 323: 1239

Yamada, H., Ono, S., Wada, S., Aoi, W., Park, E.Y., Nakamura, Y., and Sato, K. (2018). Status of food-derived glutathione in intestine, blood, and liver of rat. NPJ Sci. Food 2: 3

Yokoyama, K., Chiba, H., and Yoshikawa, M. (1992). Peptide inhibitors for angiotensin I-converting enzyme from thermolysin digest of dried bonito. Bios. Biotec. Biochem. 56: 1541-1545.

Yokozeki, K., and Abe, I. (2021). A novel route for aspartame production by combining enzymatic and chemical reactions for industrial use. Biosci. Biotech. Biochem. 85: 464-446.

Zhao, H., Zhou, J., Zhang, K., Chu, H., Liu, D., Poon, V.K.M., Chan, C.C.-S., Leung, H.-C., Fai, N., Lin, Y.-P., Zhang, A.J.-X., Jin, D.-Y., Yuen, K.-Y., and Zheng, B.-J. (2016). A novel peptide with potent and broad-spectrum antiviral activities against multiple respiratory viruses. Sci. Rep. 6 $1-13$.

Zheng, Y., Martin-Morales, A., Wang, J., Fujishima, M., Okumura, E., and Sato, K. (2021). Phenethylamine in chlorella alleviates high-fat dietinduced mouse liver damage by regulating generation of methylglyoxal. NPJ Sci. Food 5(1): 22 